\title{
Short and long-term results of sternectomy for sternal tumours
}

\author{
Stefano Bongiolatti ${ }^{1 *}$, Luca Voltolini ${ }^{1}$, Sara Borgianni ${ }^{1}$, Roberto Borrelli ${ }^{1}$, Marco Innocenti ${ }^{2}$, Giulio \\ Menichini ${ }^{2}$, Leonardo Politi ${ }^{1}$, Giorgia Tancredi ${ }^{1}$, Domenico Viggiano ${ }^{1}$, Alessandro Gonfiotti ${ }^{*}$ \\ ${ }^{1}$ Thoracic Surgery Unit, ${ }^{2}$ Plastic Surgery Unit, Careggi University Hospital, Florence, Italy \\ Contributions: (I) Conception and design: S Bongiolatti, A Gonfiotti; (II) Administrative support: S Bongiolatti; (III) Provision of study materials or \\ patients: S Bongiolatti; (IV) Collection and assembly of data: S Bongiolatti; (V) Data analysis and interpretation: S Bongiolatti, A Gonfiotti; (VI) \\ Manuscript writing: All authors; (VII) Final approval of manuscript: All authors. \\ *These authors contributed equally to this work. \\ Correspondence to: Stefano Bongiolatti, MD. Thoracic Surgery Unit, Careggi University Hospital, Largo Brambilla, 1, 50134 Florence, Italy. \\ Email: stefanobongiolatti@gmail.com.
}

Background: We analyzed our experience in sternal resections (SRs) for primary or secondary neoplasm focusing on technical aspects of reconstruction, post-operative outcomes and long term survival.

Methods: From January 2005 to December 2015, 36 patients (24 males, 67\%) underwent surgical excision of primary (chondrosarcoma $n=18$ patients, $50 \%$; osteosarcoma $n=2,6 \%$; Ewing sarcoma $n=1,3 \%$; other $\mathrm{n}=2,6 \%$ ) or secondary (breast cancer $\mathrm{n}=7,19 \%$; kidney carcinoma $\mathrm{n}=2,6 \%$ ) sternal tumour. We performed $\mathrm{n}=30$ partial sternectomy and $\mathrm{n}=6$ total sternectomy with en-bloc resection of the sternocostal cartilages in all patient and extended resection in 7 patients. Stability was obtained with prosthetic material, rigid and non-rigid and a muscular flap: rigid material [Strasbourg Thoracic Osteosynthesis System (STRATOS), MedXpert $\mathrm{GmbH}]$ and muscle flap n=11 (30.6\%); polytetrafluoroethylene patch and muscle flap n=6 (16.7\%); muscle flap alone $\mathrm{n}=19$ (52.8\%).

Results: The 30-day mortality rate was 0, overall complication rate was $19 \%$. The median ICU stay was 1.5 days and mean hospital stay was 10.6 \pm 5.9 days. We obtained a complete (R0) resection in all patients. Overall survival (OS) at 5 and 10 years were $59 \%$ and $40 \%$; in the group of primary neoplasm OS rate at 5 and 10 years was $79 \%$ and 54\%. Disease free survival (DFS) rate at 5 years was $61 \%$. Higher grading was identified as negative prognostic factor.

Conclusions: Wide radical resections of anterior chest wall are basilar in a multimodality treatment for primary or metastatic neoplasm of the sternum. Stabilization with titanium bars and clips provides rigidity of chest wall with good functional results.

Keywords: Sternectomy; chest-wall reconstruction; chest-wall prosthesis; chondrosarcoma; titanium bars and clips

Submitted Dec 22, 2016. Accepted for publication Apr 01, 2017.

doi: $10.21037 /$ jtd.2017.10.94

View this article at: http://dx.doi.org/10.21037/jtd.2017.10.94

\section{Introduction}

Sternal tumours represent an uncommon and heterogeneous group of tumours which can be divided into primary sternal tumours (PSTs) and secondary sternal tumours (SST). PSTs include primary bony or cartilaginous tumours, soft tissue sarcomas and small cell tumours (Ewing's sarcomas, plasmacytomas, and lymphomas). SSTs include metastases as well as locally invasive and recurrent cancers; among this group, breast cancer recurrence (BCR) is the most frequent and surgically treated SST.

However sternal tumours pose similar surgical and clinical problems, so that sternal resections (SRs) are usually considered together (1). During the last decade, new methods of surgical treatment and advances in techniques of rigid and musculocutaneous reconstructions have facilitated the treatment of these uncommon tumours (2-4). The reconstruction of the anterior chest wall is 
basilar to restore the integrity and rigidity of the anterior thoracic wall, to prevent respiratory impairment, to isolate the mediastinum from infections and to protect the underlying structures by trauma. Nowadays reconstruction techniques are heterogeneous and often include the use of different prosthetic materials as methylmethacrilate, polytetrafluoroethylene, titanium bars, bone homograft or allograft and musculocutaneous flap (5-11).

We retrospectively reviewed our experience with SRs in 36 patients performed during a 10 -year period, to evaluate the techniques of reconstruction, early clinical outcomes and survival.

\section{Methods}

From January 2005 to December 2015, we retrospectively reviewed the medical records of all patients underwent sternectomy for primary or secondary neoplasm, different from lung cancer, involving the sternum, costochondral cartilages, anterior ribs, parasternal soft tissue or a combination of these structures.

Our institutional review board granted approval (2014.0317) and waived the requirement for specific informed consent for this retrospective study.

\section{Pre-operative assessment}

Diagnostic work-up included physical examination, standard chest-X ray, computed tomography (CT) scan in all cases to delineate the bone, soft tissue, mediastinal and pleural extension of the cancer; positron emission tomography-CT (PET-CT) scan was used to exclude a disseminated disease. In suspicion of thoracic outlet, mediastinal or neurovascular involvement, we used also magnetic resonance (MR). In case of a planned pedicled skin or muscle flap, a Doppler ultrasound evaluation of the flap feeding-vessels was always performed.

Where the histological diagnosis was not present at the time of our observation, we performed a core-needle aspiration or an excisional biopsy. The need to perform induction chemotherapy or postoperative chemotherapy and radiotherapy in case of high-grade sarcomas was discussed and planned with the medical oncologist and the radiotherapist in a multidisciplinary institutional Sarcoma Group (orthopaedic oncology, medical adult and paediatric oncology, radiotherapy, pathologist, radiology). Neoadjuvant therapy was considered in case of metastatic cancer and high-risk sarcomas such as high-grade soft tissue sarcomas and high-risk bony sarcomas (osteosarcoma,
Ewing sarcoma, and mesenchymal sarcoma).

Most of our cases were planned and performed by a team of thoracic surgeons and a plastic surgeon. In all cases, the thoracic surgeon performed resection and rigid or non-rigid reconstruction, then the plastic surgeon performed softtissue repair of the defect.

\section{Surgical technique}

Our surgical policy in treating sternal tumours was, in general, as follows: skin incision always included the site of the previous biopsy, the invaded skin or previously irradiated tissues; in case of PST resection included at least $3 \mathrm{~cm}$ of free margins, the adjacent sternocostal cartilages and the affected ribs in case of sternocostal lesion; in case of SSTs free margins $<3 \mathrm{~cm}$ were considerate adequate; tumour extension into the chest cavity was evaluated and any other structure involved in the tumour was also excised. The extent of SR depended on the dimension and the location of the tumour and was defined on the basis of the longitudinal sternal diameter as it follows: $<90 \%$ partial sternectomy; $\geq 90 \%$ subtotal; $100 \%$ total (12).

Surgical margins were classified into wide, marginal, and intralesional using the Enneking classification (12) as adapted to the chest wall by the Scandinavian sarcoma pathology group (13): (I) Type 1: wide surgical margin (intact pleura internally, intact muscle fascia externally, transverse rib resection $>2 \mathrm{~cm}$ from the tumour on both sides); (II) Type 2: macroscopic and microscopic free from tumour but $<2 \mathrm{~cm}$ distance); (III) Type 3: intralesional (lesion macroscopically taken out in pieces or microscopic positive margins). Pathological specimens were analysed by expert pathologists and chondrosarcomas were classified as grade 1 to 3 (14).

En-bloc resection and reconstruction were performed as a one-stage procedure in all cases. Reconstruction included the use of prosthetic reconstruction when needed (rigid and/or non-rigid) and muscle or musculocutaneous flap, in order to achieve the chest wall stability, to cover the defect and protect the mediastinum. Regarding the indications of reconstruction, when the oncological resection caused a wide defect (area approximately larger than $5 \mathrm{~cm} \times 5 \mathrm{~cm}$ or excision of more than two ribs and sternum), then is necessary to restore the integrity of the chest wall using a rigid material. This circumstance is evaluated preoperatively and intra-operatively by the surgical staff. Also the aesthetic result is considered, mostly if the defect can cause a paradoxical respiratory movement. 
All patients were extubated immediately after surgery and transferred to the intensive care unit (ICU) for intensive monitoring. Since the immediate post-operative period patients were involved in a respiratory physiotherapy program.

\section{Statistical analysis}

Statistical analysis was performed using SPSS 16.0 software (SPSS Inc., Chicago, IL, USA). Continuous variables are expressed as median, mean values $\pm \mathrm{SD}$ and compared with unpaired $t$-test. Categorical variables were analyzed using $\chi$-square test. The Kaplan-Meier method was used to calculate overall and disease-free survival. Overall survival (OS) was calculated from the date of operation to death or date of the last follow-up (December 2015); disease-free survival was calculated for those patients who received a radical resection from the date of operation to the date of the first evidence of recurrence. Differences in overall and disease-free survival were determined by log-rank analysis. Multivariable analysis was performed to identify risk factors and the following variables were evaluated for prognostic significance: age, gender, histology (PSTs vs. SSTs), grade, margin status. Significance was defined as $\mathrm{P}<0.05$.

\section{Results}

From January 2005 to December 2015, 36 consecutive patients (24 males, $67 \%$ ) underwent surgical excision of primary (23 patients, $64 \%$ ) or secondary (13 patients, $36 \%$ ) sternal tumour. Demographical, pre-operative and histology data are depicted in Table 1.

Tumours location was as it follows: upper thirdmanubrium $\mathrm{n}=9(25 \%)$, upper third-sternoclavicular junction $\mathrm{n}=2(5.5 \%)$, middle third-sternal body, $\mathrm{n}=21$ $(58.5 \%)$, sternal body-lower third $n=4(11 \%)$.

\section{Extent of resection}

Characteristics of resections are showed in Table 2 and in the schematic Figure 1. We performed 30 (83\%) partial sternectomies and $6(17 \%)$ total/subtotal sternectomies. In 9 cases the tumour involved the upper part of the sternum and resection of the manubrium en-bloc with the middle third of clavicles was needed to achieve $<3 \mathrm{~cm}$ free margins. A median of 3 (range, 1-6) adjacent condrocostal cartilages was removed. Resections were extended to the adjacent structures in 7 patients (19\%): $n=5$ (14\%) lung wedge resection, $\mathrm{n}=3$ (8\%) partial resection of diaphragm, 1 (3\%) partial resection of the pericardium, $1(3 \%)$ resection of the subclavian vein (Table 2). In $\mathrm{n}=2$ patients with a wide sternoclavicular joint lesion, the clavicle was completely removed together with the manubrium and the upper third of the sternal body.

\section{Reconstruction}

All sternectomies underwent reconstruction, for both, cosmetic and functional reasons. Type of reconstructions is resumed in Table 2. In most partial sternectomies, reconstruction consisted in bilateral pectoralis major myocutaneous advancement flaps ( $\mathrm{n}=19,52.7 \%)$.

All the remaining 17 (47\%) cases we performed a prosthetic reconstruction using expanded-polytetrafluoroethylene (ePTFE) $2 \mathrm{~mm}$ thick patch (Dual Mesh plus, W.L. Gore \& Assoc, Flagstaff, Az, USA) (Figure 2) associated with muscle flap in $\mathrm{n}=6(16.7 \%)$ patients (Figures 3 and 4 ) or a combination of rigid titanium bars and rib clips $(8,9,11)$ [Strasbourg Thoracic Osteosynthesis System (STRATOS) MedXpert $\mathrm{GmbH}$ ] combined with muscle flap and/or PTFE in $\mathrm{n}=11(30.5 \%)$ patients. In this group a pedicled muscle or musculocutaneous flap was needed in $n=5 / 17$ (29\%) cases, in the remaining patients we covered the prosthesis with pectoralis major myocutaneous advancement flaps. In one case of total sternectomy for a wide and ulcerated PST, a delayed skin grafting was necessary after a combined reconstruction with STRATOS and a pedicled left latissimus dorsi muscle flap. The type of muscle flap was chosen by plastic surgeon on the basis of soft tissues local condition (i.e., previous surgery or radiation therapy) and on the pre-operative Doppler ultrasound evaluation of flap feeding-vessels.

\section{Post-operative results}

A radical resection (type 1 margins) was accomplished in 31 (86\%) patients. We had 5 (14\%) type 2 margins but no patients presented an intralesional resection. Histological examination of chondrosarcomas demonstrated G1 tumour in $11(61 \%)$ patients, G2 in $6(33.3 \%)$ and $\mathrm{G} 3$ in $1(5.6 \%)$ patients (Table 1).

Post-operative mortality was zero. Post-operative and in-hospital complications are depicted in Table 3. One patient required prolonged mechanical ventilation and tracheostomy secondary to a pulmonary infection; we observed also $\mathrm{n}=1$ bleeding (from the muscle flap), requiring blood transfusions and $n=1$ pneumonia. Two patients (5.6\%) 
Table 1 Demographical, pre-operative and histologic data

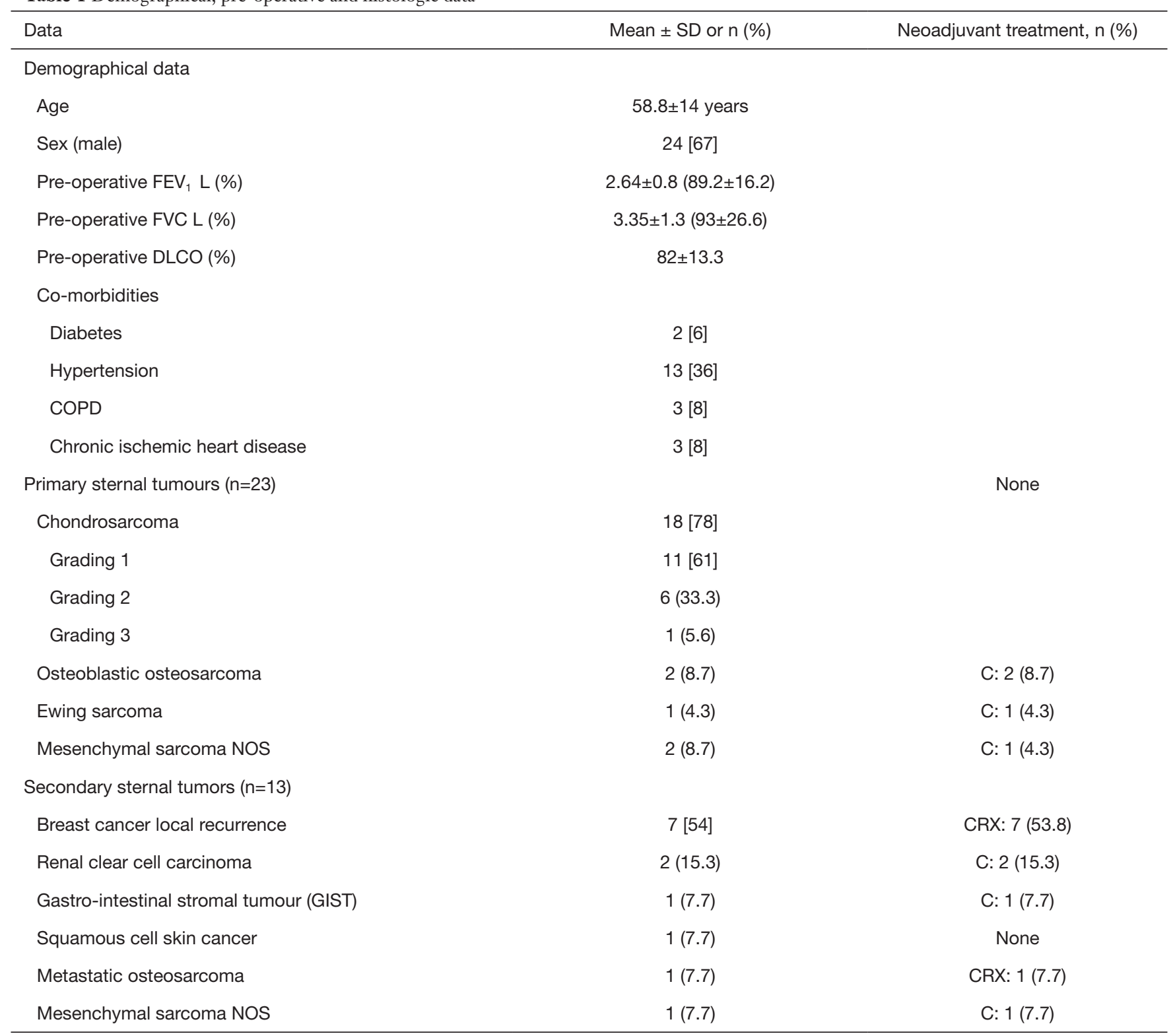

$\mathrm{FEV}_{1}$, forced expiratory volume in 1 second; FVC, forced vital capacity; DLCO, diffusing capacity of the lung for carbon monoxide; COPD, chronic obstructive pulmonary disease; C, chemotherapy; CRX, chemoradiation therapy; NOS, not otherwise specified.

developed wound infection: one case successfully treated with Vacuum Assisted Closure (VAC) therapy; the second requiring a surgical revision with mesh removal and new reconstruction with a double muscle flap (latissimus dorsi and contralateral pectoralis major).

About results of rigid reconstruction, we experienced a symptomatic bars displacement in $\mathrm{n}=1$ (9\%) case, 3 months after the discharge; the fractured and dislocated bar was in contact with the pericardium, causing arrhythmia and chest pain. The patient underwent surgical removal of the bars and re-reconstruction with muscle flap, obtaining a good cosmetic and mechanical result.

\section{Oncological and long-term outcomes}

At a median follow-up of 24 months (range, 6-132 months) $22(61 \%)$ patients were alive. We had local recurrence in 2 patients of group of PST (8\%) at a median of 15 months. 
Table 2 Extent of resection and reconstruction (N=36)

\begin{tabular}{|c|c|}
\hline Resection and reconstruction & n (\%) \\
\hline \multicolumn{2}{|l|}{ Surgical resection } \\
\hline Partial sternectomy & $30[83]$ \\
\hline Total/subtotal sternectomy & $6[17]$ \\
\hline Lung wedge & $5[14]$ \\
\hline Diaphragm & $3[8]$ \\
\hline Pericardium & $1[3]$ \\
\hline Subclavian vein & $1[3]$ \\
\hline Clavicle & $2[6]$ \\
\hline \multicolumn{2}{|l|}{ Reconstruction } \\
\hline Muscular flap & $19(52.8)$ \\
\hline \multicolumn{2}{|l|}{ Pectoralis major } \\
\hline ePTFE + mucolar flap & 5 (13.9) \\
\hline Pectoralis major & $4(11.1)$ \\
\hline Latissimus dorsi & $1(2.8)$ \\
\hline ePTFE+ musculocutaneous flap & $1(2.8)$ \\
\hline \multicolumn{2}{|l|}{ Latissimus dorsi } \\
\hline Titanium bars + muscular flap & $6(16.7)$ \\
\hline Pectoralis major & $4(11.1)$ \\
\hline Rectus abdominis & $1(2.8)$ \\
\hline Latissimus dorsi & $1(2.8)$ \\
\hline Titanium bars + ePTFE + muscular flap & $5(13.9)$ \\
\hline Pectoralis major & $4(11.1)$ \\
\hline Latissimus dorsi & $1(2.8)$ \\
\hline
\end{tabular}

ePTFE, expanded-polytetrafluoroethylene.

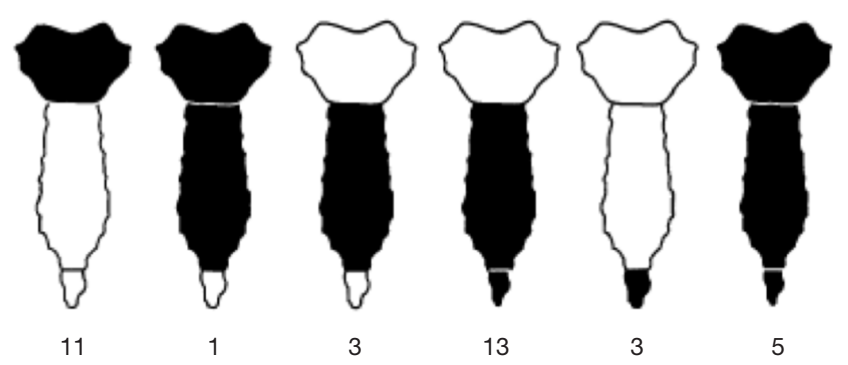

Figure 1 Schema of the extent of resection.

All patients with local recurrence underwent a new resection after a new medical oncology treatment, in case of histology different from chondrosarcoma.
Systemic disease in PST occurred in 5 patients $(21 \%)$ and the most common site of metastasis was the lung $(\mathrm{n}=4$, $17 \%)$ and mediastinal lymph nodes $(\mathrm{n}=1,4 \%)$. Adjuvant treatment was administered in case of relapse and after SR of osteosarcomas and Ewing sarcoma.

OS at 5 and 10 years was $59 \%$ and $40 \%$ (Figure 5 ) respectively. In the group of PSTs, the overall 5- and 10-year survival was $79 \%$ and $54 \%$ respectively (Figure 6 ) and disease free survival (DFS) rate at 5-year on radical resections was $61 \%$ (Figure 7).

In patients who underwent sternectomy for secondary lesions, the 5- and 10-year survival were statistically worse compared with PSTs at univariate analysis (log rank test $\mathrm{P}<0.01$ ) (Figure 6).

Patients radically treated for PSTs $(\mathrm{P}<0.01)$ with wide margins have a favourable outcome (Figures 6 and 8 ). Furthermore, patients operated for low-grade chondrosarcomas have a favourable outcome $(\mathrm{P}<0.01)$ (Figure 9). All oncological results are resumed in the Table 4. The independent predictor of survival was grading $(\mathrm{P}=0.049)$ as depicted in Table 5. In univariate analysis regarding DFS a low grade chondrosarcoma (data not showed), radically treated with wide margins $(\mathrm{P}=0.03)$ lead to a favourable prognosis, but only the radicality of resection with type 1 margins $(\mathrm{P}=0.02)$ was identified as an independent prognostic factor at multivariable analysis (Table 5).

\section{Discussion}

The sternum can be interested by primary neoplasm, by metastatic osteotropic tumours or by local recurrence from breast or skin cancer.

PSTs and SSTs are rare, accounting for less than $30 \%$ of all chest wall tumours (3). The most common origin of PST is bone or cartilage, even if some authors report an equal distribution between bone, cartilage and soft tissues $(1,4)$. Sternectomy is the treatment of choice for PSTs because these tumours are often chemo- and radio-insensitive like chondrosarcomas, while in case of SSTs the SR is indicated with palliative intent (control of pain, removal of ulcerating lesion and debulking) (1) such as in case of BCR. Tenets of SRs and reconstructions consist in an adequate radical resection associated with the maintenance of chest stability, lung function and an acceptable cosmetic result (15). Consequently, a critical point, common to all wide anterior chest-wall resections, is a suitable prosthetic replacement, able to restore the rigidity of the chest and to prevent paradoxical motion, and a healthy soft-tissue coverage 

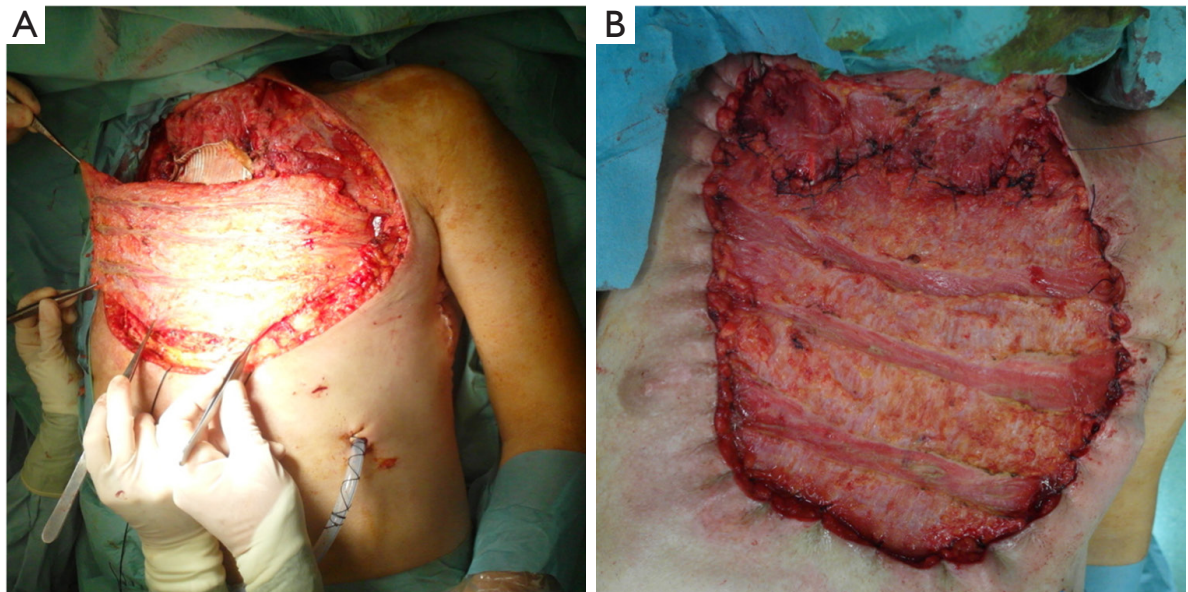

Figure 2 Intraoperative image of resection of the manubrium and reconstruction with ePTFE convered with latissimus dorsi muscle flap. ePTFE, expanded-polytetrafluoroethylene.
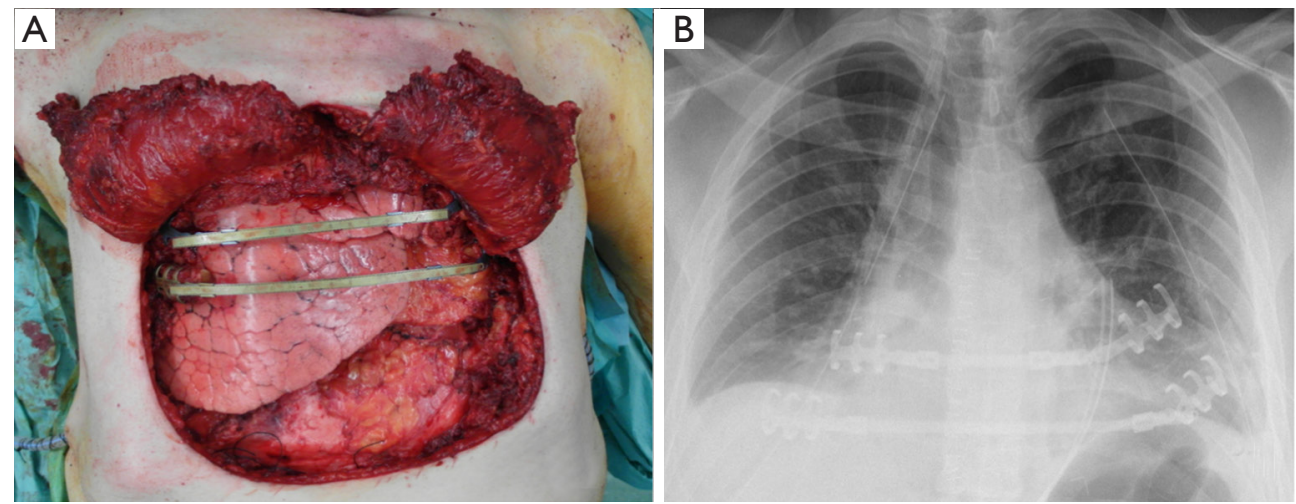

Figure 3 (A) Intraoperative images of lower-third partial sternectomy for chondrosarcoma, rigid reconstruction with STRATOS and coverage with sliding PM flap; (B) chest X-ray in postoperative day 7 of the same patient. STRATOS, Strasbourg Thoracic Osteosynthesis System.
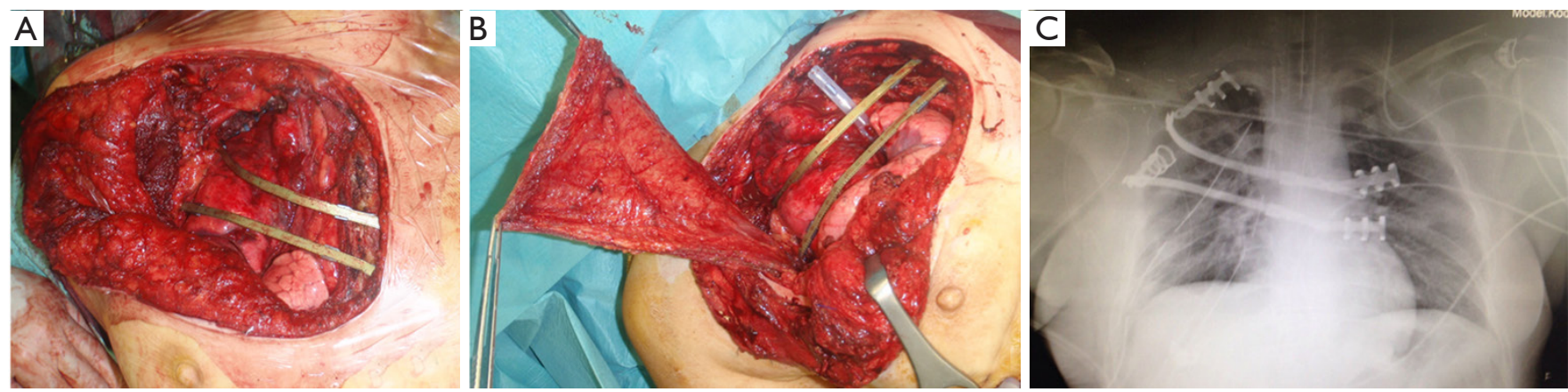

Figure 4 (A) Intraoperative images of upper-third partial sternectomy associated with resection of II, III and IV costal cartilage for chondrosarcoma and reconstruction with STRATOS covered by latissimus dorsi muscle flap (B). Chest X-ray in postoperative day 1 of the same patient (C). STRATOS, Strasbourg Thoracic Osteosynthesis System. 
Table 3 Operative and post-operative data

\begin{tabular}{lc}
\hline Data & Value \\
\hline Operative time & $310 \pm 118$ \\
& {$[120-530]$ min } \\
ICU stay & $1.5 \pm 0.7[1-15]$ days \\
Hospital stay & $10.6 \pm 5.9[5-33]$ days \\
Complications & $7 / 36(19.4 \%)$ \\
Pneumonia & $2(5.6 \%)$ \\
Respiratory failure requiring mechanical & $1(2.8 \%)$ \\
ventilation & \\
Atrial fibrillation & $1(2.8 \%)$ \\
Bleeding requiring blood transfusion & $1(2.8 \%)$ \\
Flap or wound infection & $2(5.6 \%)$ \\
Radical resection (type 1 margin) & $31 / 36(86 \%)$ \\
Post-operative FEV ${ }_{1}$ & $2.24 \mathrm{~L}(75 \%)$ \\
Post-operative FVC & $2.85 \mathrm{~L}(79 \%)$ \\
Post-operative DLCO\% & $69.7 \%$ \\
Local recurrence & $2 / 23(8.7 \%)$ \\
Distant recurrence & $5 / 23(21.7 \%)$ \\
Follow-up & $24.5[6-132] \mathrm{months}$ \\
\hline ICU, intensive care unit; FEV ${ }_{1}$, forced expiratory volume in 1 \\
the lung for carbon monoxide. & \\
& \\
second; FVC, forced vital capacity; DLCO, diffusing capacity of
\end{tabular}

able to seal the pleural space, to protect the mediastinum and to prevent infections $(5,15,16)$. The importance of a radical surgery has been demonstrated by several authors $(5,13,15,16)$ : by intraoperative examination of soft tissue margin and the limit of $2 \mathrm{~cm}$ on the bone, in our series, we obtained a radical resection in all cases of PST; however, in case of SST we considered as acceptable a marginal resection, but always excluding an intralesional resection. As a result, all the operations were margin free, minimizing the incidence of local recurrence ( $8 \%$ in our series).

As a wide resection of the anterior chest wall often threatens stability, reconstruction plays a crucial role in determining postoperative morbidity and mortality $(2,7,15,17)$ particularly in SRs.

Rigidity and protection of the viscera can be achieved using rigid prosthetic materials, while non-rigid materials are useful for sealing the dead space. Several reports (7-10) suggest that stabilizing the anterior chest wall with rigid material could decrease the risk of developing an acute restrictive disease, avoiding deformities and restoring an acceptable thorax volume and this leads to a reduced incidence of complication and mortality (10).

In our experience, in case of partial sternectomy ( $<90 \%$ longitudinal diameter), when a part of the manubrium or of the lower sternal body is conserved, a rigid reconstruction is not needed for functional reasons, but just for cosmetic results; these defects can be replaced just by non-rigid prostheses or muscle-flap. In case of total sternectomy, a rigid reconstruction is mandatory to maintain the rib cage respiratory function. In the last decade, for rigid reconstruction we always used STRATOS $(8,9,11)$ obtaining a low rate of overall and respiratory complications and a low rate of prosthesis or bars problems. This series confirmed that STRATOS allows a firm reconstruction after sternectomies, since it is simple to handle and to attach to the resected edges of the ribs, so that the bars are not easily dislodged. The implantation of titanium is safe and is not associated with the onset of a restrictive pattern nor a higher incidence of prosthesis related complications. In our series, we had only one symptomatic bars displacement (1/11, 9\%), occurrence similar to the first series published by Berthet et al. (9). During the follow-up, the same author noted that the implant failure is quite high $(44.4 \%)$, but without severe complications and often the rupture or the dislocation were asymptomatic. They identified the anterior location of chest wall resection and the presence of three or more implant as a significant risk factors of failure (18). Similarly other titanium rigid reconstruction systems are effectively used and reported in literature (6).

As non-rigid prosthetic materials, the most commonly used are Prolene or Marlex mesh and ePTFE $(5,7,9,15,16)$ and no significant difference has been demonstrated in the postoperative outcome or in complication rate between them (7). We used ePTFE $(n=11 ; 31 \%)$ with a low rate of complications and a good final results.

Once the chest wall has been stabilized, a soft-tissue coverage can be used to complete the reconstruction, obliterate the dead space, cover and separate the synthetic material. Actually, the major drawback of all prosthesis implant is the increased risk of infection, reported to occur in $8-20 \%(17)$. We had two cases of wound infection, one of whom happened in a high risk condition, such as a neoplastic skin ulceration is; only this case needed a new operation and removal of the ePTFE. This low rate of wound and prosthesis infection $(6 \%, 2 / 36)$ could be explained by the use of muscle or musculocutaneous flap in almost all patients; the soft tissue transfer was always 


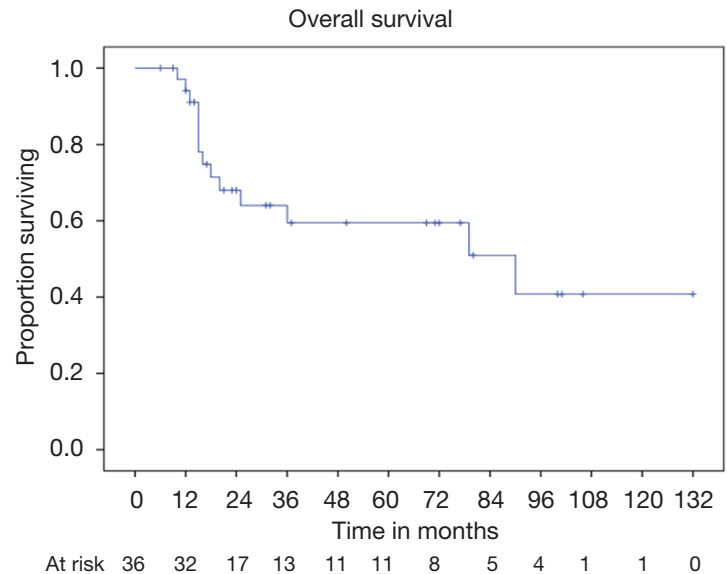

Figure 5 Overall 5- and 10-year survival curve.

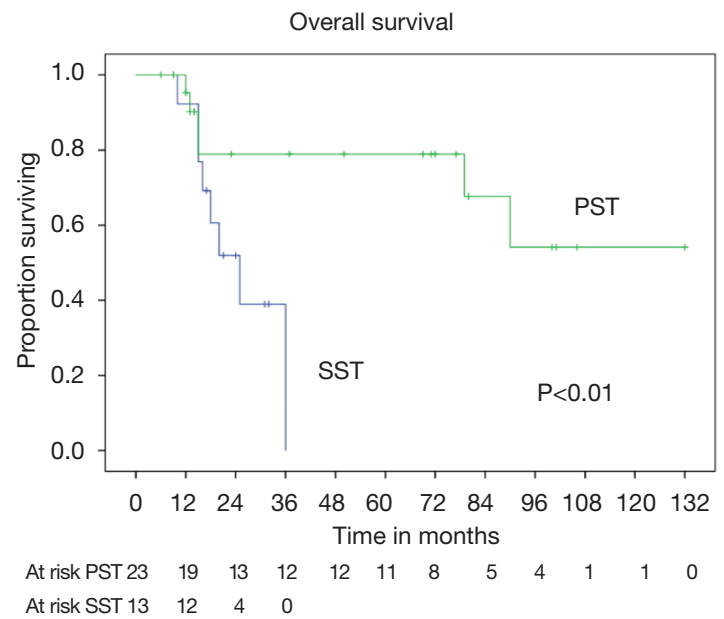

Figure 6 Outcome of patients with primary or secondary sternal tumour, the 5-year overall survival for PSTs was 79\%. PSTs, primary sternal tumours; SST, secondary sternal tumour.

performed in close interaction with a plastic surgeon, involved in the preoperative selection of the flap as well as in the surgical procedure. PM is the most frequently selected muscle for sternectomy, because of its proximity, reliability and versatility and it can be used unilaterally or bilaterally, with skin advancement or as a musculocutaneous flap $(8,9)$; we used a different muscle flap in case PM was unavailable such as for tumour infiltration or previous irradiation or surgery. In that cases, a pedicled LD flap was the preferred choice, since LD is easily transposed on thoracodorsal vessels and provides extensive coverage $(5-7,10,15,16)$.

Our study showed a low incidence of overall (19\%) and respiratory complications also in extended resections $(3 / 36,8 \%)$.

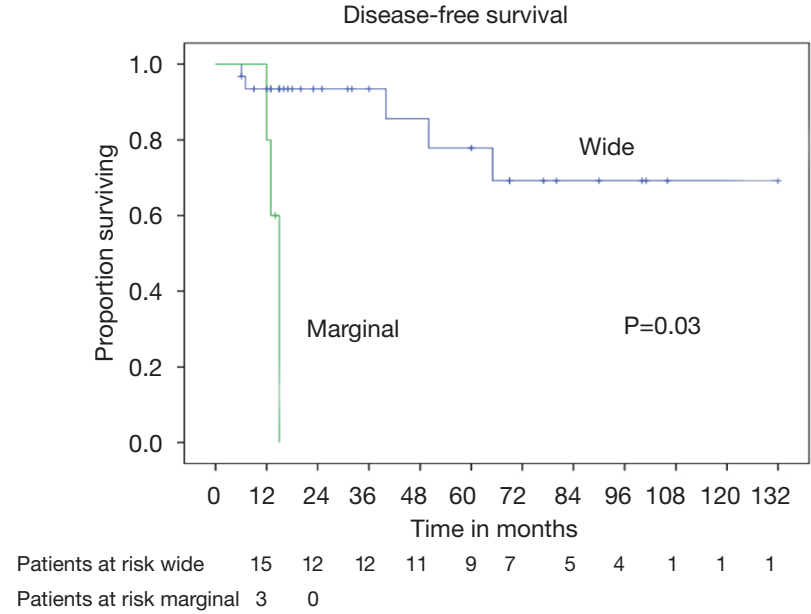

Figure 7 Disease-free survival curve based on resectional status.

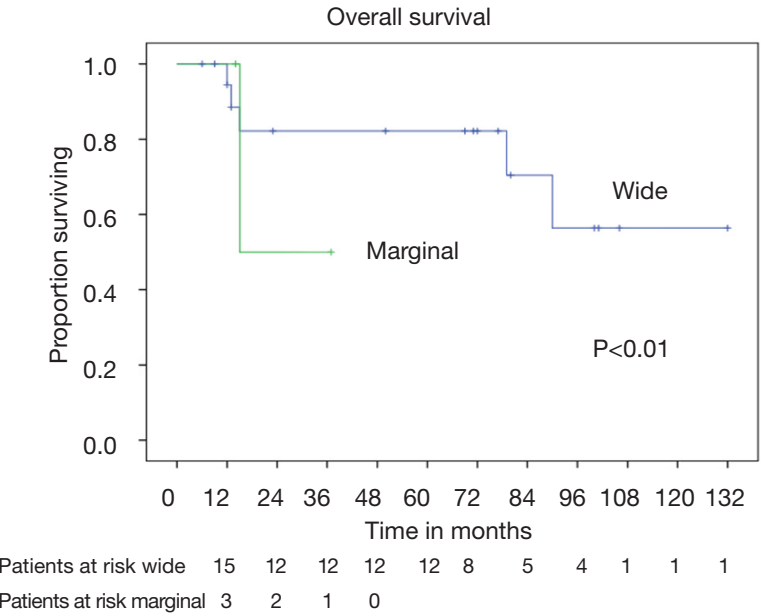

Figure 8 Survival of PST curve based on resectional status. PST, primary sternal tumour.

The 30-day mortality rate for both, total and partial SR is zero and this finding is consistent with previous published data $(0-9.5 \%)$ (3). Respiratory complications are the main cause of in-hospital mortality in chest wall resections (2), based on an impaired rib cage mechanical function, causing atelectasis, pneumonia and subsequent respiratory failure and sepsis. It is unclear whether surgical procedure and type of reconstruction could minimize the possibility of development of pulmonary complications; however, our results demonstrated that an effective rigid reconstruction, an early extubation and mobilization with an aggressive physiotherapy may decrease the incidence of respiratory impairment and related conditions. 


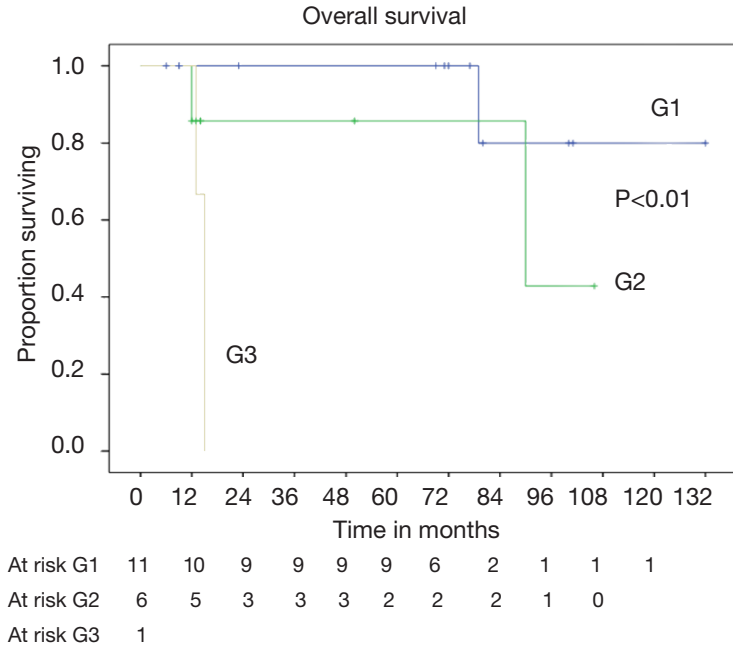

Figure 9 Survival curves based on histological grading of chondrosarcoma.

The importance of a centralized treatment in order to receive a multidisciplinary approach in chest wall tumours is widely accepted $(13,15)$; in our series every treatment has been discussed and planned in an institutional sarcoma group, composed by orthopaedic oncologist, plastic surgeon, medical oncologist and radiotherapist. As result of this co-operation, $17 \%$ and $43 \%$ of patients in this series underwent induction and adjuvant therapy, respectively. The use of induction therapies still remains controversial for low-grade PST, but different chemo or chemoradio-regimens are available for osteosarcomas, Ewing sarcomas and high-grade soft tissue sarcomas with improvement of OS $(1,15,19,20)$. Kachroo and co-workers (21) reported a higher percentage of patients (72\%) affected by chest wall soft tissue sarcoma treated by induction therapy and subsequent surgical resection without higher incidence of complication or mortality, obtaining a 5 -year OS rate of $66 \%$ and a 5 -year DFS rate of $47 \%$. These results confirmed the non-responsiveness of lowgrade sarcomatous tumours to chemotherapy and that radical resection provides better long-term outcomes.

The 5-year OS after SR for malignancy depends on histology and has been reported between $50-80 \%$ for PSTs $(19,20)$ and $20-40 \%$ for RBC (3). In our series, after a median follow-up or 24 months, we observed an overall 5 -year survival of $59 \%$. In our experience, the 5 -year survival ranges very widely from $79 \%$ of chondrosarcomas to $0 \%$ of SSTs.

The most common tumour of the anterior thoracic wall is chondrosarcoma, characterized by a slow growth and a tendency to local infiltration; since its chemo-resistance and a low radio-sensitivity, a complete full-thickness resection is still the best treatment option. A radical resection without adjuvant therapy is associated with long term survival and OS ranged from $80 \%$ to $60 \%$ at 5 years $(1-4,13)$. In our series, we found a $79 \%$ and $54 \%$ of overall 5 and 10 years survival respectively for PST, most of which were chondrosarcomas (18/36, $50 \%$ of all series).

The role of the free margin resection is widely discussed $(12,13)$ and we considered safe a margin of $3 \mathrm{~cm}$ away from the sarcomatous lesion, while for SSTs we considered adequate a distance less to $3 \mathrm{~cm}$. In the large populationbased study of Scandinavian Sarcoma Group, is clearly demonstrated the influence of wide free margins on survival; patients radically treated have higher probability of survival at 10 years (92\%) compared with intralesional resections (13). Also others authors previously showed a better outcome in term of OS for patients surgically treated without margin involvement $(1,19,22)$. In our report, the DFS is negatively influenced by margins; actually patients with incomplete resection developed early local or distant recurrence. However, a complete resection was not identified as prognostic factor, since the differences in terms of survival have been not significant due to the small population study with a low incidence of type 2 margins $(3 / 23,13 \%)$. The patients with local recurrence from PST, considered fit for surgery, underwent re-resection rather than adjuvant radiotherapy; the use of adjuvant radiation did not seem to have a clear association to decrease local recurrence, moreover van Geel (20) described an extensive use of postoperative radiation, but reported an overall local recurrence rate of $33 \%$ and a significative reduction of OS. Other positive prognostic factors on OS, identified in large series, are the histological low grading $(1,6,21)$, the completeness of resection $(1,6,19,23)$, younger age and no adjuvant treatment $(6,20)$. For chondrosarcomas, we identified the histological grading as a prognostic factor on OS and DFS. Grading is a well-accepted prognostic factor in bone and soft-tissue sarcoma surgery and we could expect this result.

Our study has some limitations: (I) is a single institution series, retrospectively analyzed between 2005-2015; (II) the patients cohort is heterogeneous about the sternal neoplastic involvement and the reconstruction technique; (III) there is a lack of a structured follow-up investigation about cosmetic outcome, daily activity level and quality of life; (IV) the predictors of OS and DFS should be interpreted with caution because the study population is small due to the rarity of PST and therefore it would be desirable to perform multi-institutional studies that could 
Table 4 Survival table and univariate analysis of prognostic factor (log rank test)

\begin{tabular}{|c|c|c|c|c|c|c|}
\hline Variable & 5-year OS, \% & 10-year OS, \% & Mean & SE & $95 \% \mathrm{Cl}$ & $P$ value \\
\hline DFS in PST & 61 & - & 80.46 & 10.89 & $59.11-101.8$ & - \\
\hline PST & 79 & 54 & 95.46 & 11.92 & $72.09-118.83$ & 0.019 \\
\hline SST & 0 & - & 24.86 & 3.02 & $18.93-30.78$ & \\
\hline Chondrosarcoma G2 & 83 & 0 & 87.25 & 11.20 & $65.28-109.21$ & \\
\hline Chondrosarcoma G3 & 0 & - & 14.33 & 10.66 & $13.02-15.04$ & \\
\hline Wide resection & 82 & 56 & 98.76 & 12.06 & $75.11-122.42$ & 0.50 \\
\hline Marginal resection & 0 & 0 & 26 & 7.77 & $10.75-41.24$ & \\
\hline Age $<65$ years & 58 & 28 & 62.76 & 9.07 & $44.96-80.55$ & \\
\hline
\end{tabular}

OS, overall survival; SE, standard error; CI, confidence interval; DFS, disease-free survival; PST, primary sternal tumour; SST, secondary sternal tumour.

Table 5 Prognostic factors for overall survival (OS) and disease free survival (DFS) in PSTs group: multivariable Cox analysis

\begin{tabular}{|c|c|c|c|c|}
\hline Variable & \multicolumn{2}{|l|}{ OS } & \multicolumn{2}{|l|}{ DFS } \\
\hline Gender: male vs. female & $1.800(0.327-9.9)$ & 0.49 & $2.472(0.0446-13.702)$ & 0.30 \\
\hline Age: $>65$ vs. $<65$ years & $1.211(0.237-6.182)$ & 0.81 & $0.295(0.034-2.538)$ & 0.26 \\
\hline Margin: type 2 vs. type 1 & $2.091(0.217-20.15)$ & 0.52 & $16.296(1.410-188.274)$ & 0.021 \\
\hline
\end{tabular}

PST, primary sternal tumour.

clarify the role of neoadjuvant and adjuvant therapies.

In conclusion, SR is a safe surgical procedure with low mortality and low complication rate. An institutional multidisciplinary approach is confirmed as the best option in planning a multimodality treatment, in order to achieve the best early and long term results. The reconstruction technique with titanium bars and rib clips represents a reliable rigid reconstructive option, with low rate of equipment failures and good results on chest wall mechanics. Our study confirms that low-grade sternal sarcomas, radically treated, are associated with long-term survival.

\section{Acknowledgements}

None.

\section{Footnote}

Conflicts of Interest: Presented at 30th EACTS Annual Meeting, 1-5 October 2016, Barcelona. Session: Chest wall and mediastinum.

Ethical Statement: Our institutional review board granted approval (2014.0317) and waived the requirement for specific informed consent for this retrospective study.

\section{References}

1. Incarbone $M$, Nava $M$, Lequaglie C, et al. Sternal resection for primary or secondary tumors. J Thorac Cardiovasc Surg 1997;114:93-9. 
2. Chapelier AR, Missana MC, Couturaud B, F et al. Sternal resection and reconstruction for primary malignant tumors. Ann Thorac Surg 2004;77:1001-6; discussion 1006-7.

3. Koppert LB, van Geel AN, Lans TE, et al. Sternal resection for sarcoma, recurrent breast cancer, and radiation-induced necrosis. Ann Thorac Surg 2010;90:1102-1108.e2.

4. Soysal O, Walsh GL, Nesbitt JC, et al. Resection of sternal tumors: extent, reconstruction, and survival. Ann Thorac Surg 1995;60:1353-8; discussion 1358-9.

5. Chapelier AP, Macchiarini P, Rietjens M, et al. Chest wall reconstruction following resection of large primary malignant tumours Eur J Cardiothorac Surg 1994;8:351-6; discussion 357.

6. Marulli G, Duranti L, Cardillo G, et al. Primary chest wall chondrosarcomas: results of surgical resection and analysis of prognostic factors. Eur J Cardiothorac Surg 2014;45:e194-201.

7. Weyant MJ, Bains MS, Venkatraman E, et al. Results of chest wall resection and reconstruction with and without rigid prosthesis. Ann Thorac Surg 2006;81:279-85.

8. Fabre D, El Batti S, Singhal S, et al. A paradigm shift for sternal reconstruction using a novel titanium rib bridge system following oncological resections. Eur J Cardiothorac Surg 2012;42:965-70.

9. Berthet JP, Canaud L, D'Annoville T, et al. Titanium plates and Dualmesh: a modern combination for reconstructing very large chest wall defects. Ann Thorac Surg 2011;96:1709-16.

10. Lardinois D, Müller M, Furrer M, et al. Functional assessment of chest wall integrity after methylmethacrylate reconstruction. Ann Thorac Surg 2000;69:919-23.

11. Gonfiotti A, Santini PF, Campanacci D, et al. Use of moldable titanium bars and rib clips for total sternal replacement: a new composite technique. J Thorac Cardiovasc Surg 2009;138:1248-50.

12. Enneking WF, Spanier SS, Goodman MA. A system for the surgical staging of musculoskeletal sarcoma. Clin Orthop Relat Res 1980;(153):106-20.

Cite this article as: Bongiolatti S, Voltolini L, Borgianni S, Borrelli R, Innocenti M, Menichini G, Politi L, Tancredi G, Viggiano D, Gonfiotti A. Short and long-term results of sternectomy for sternal tumours. J Thorac Dis 2017;9(11):43364346. doi: 10.21037/jtd.2017.10.94
13. Widhe B, Bauer HC; Scandinavian Sarcoma Group. Surgical treatment is decisive for outcome in chondrosarcoma of the chest wall: a population-based Scandinavian Sarcoma Group study of 106 patients. J Thorac Cardiovasc Surg 2009;137:610-4.

14. Evans HL, Ayala AG, Romsdahl MM. Prognostic factors in chondrosarcoma of bone: a clinicopathologic analysis with emphasis on histologic grading. Cancer 1977;40:818-31.

15. Walsh GL, Davis BM, Swisher SG, et al. A singleinstitutional, multidisciplinary approach to primary sarcomas involving the chest wall requiring full-thickness resections. J Thorac Cardiovasc Surg 2001;121:48-60.

16. Gonfiotti A, Santini PF, Campanacci D, et al. Malignant primary chest-wall tumours: techniques of reconstruction and survival. Eur J Cardiothorac Surg 2010;38:39-45.

17. Mansour KA, Thourani VH, Losken A, et al. Chest wall resections and reconstruction: a 25-year experience. Ann Thorac Surg 2002;73:1720-5; discussion 1725-6.

18. Berthet JP, Gomez Caro A, Solovei L, et al. Titanium Implant Failure After Chest Wall Osteosynthesis. Ann Thorac Surg 2015;99:1945-52.

19. Martini N, Huvos AG, Burt ME, et al. Predictors of survival in malignant tumors of the sternum. J Thorac Cardiovasc Surg 1996;111:96-105; discussion 105-6.

20. van Geel AN, Wouters MW, Lans TE, et al. Chest wall resection for adult soft tissue sarcomas and chondrosarcomas: analysis of prognostic factors. World J Surg 2011;35:63-9.

21. Kachroo P, Pak PS, Sandha HS, et al. Single-institution, multidisciplinary experience with surgical resection of primary chest wall sarcomas. J Thorac Oncol 2012;7:552-8.

22. Butterworth JA, Garvey PB, Baumann DP, et al. Optimizing reconstruction of oncologic sternectomy defects based on surgical outcomes. J Am Coll Surg 2013;217:306-16.

23. Ahmad U, Yang H, Sima C, et al. Resection of Primary and Secondary Tumors of the Sternum: An Analysis of Prognostic Variables. Ann Thorac Surg 2015;100:215-21; discussion 221-2. 